This item was submitted to Loughborough's Research Repository by the author.

Items in Figshare are protected by copyright, with all rights reserved, unless otherwise indicated.

\title{
Variables in dollar terms versus in rate terms: The case of market feedback on merger negotiations
}

PLEASE CITE THE PUBLISHED VERSION

http://dx.doi.org/10.1016/j.irfa.2017.01.002

PUBLISHER

(C) Elsevier

VERSION

AM (Accepted Manuscript)

\section{PUBLISHER STATEMENT}

This work is made available according to the conditions of the Creative Commons Attribution-NonCommercialNoDerivatives 4.0 International (CC BY-NC-ND 4.0) licence. Full details of this licence are available at: https://creativecommons.org/licenses/by-nc-nd/4.0/

\section{LICENCE}

CC BY-NC-ND 4.0

\section{REPOSITORY RECORD}

Chou, Hsin-I, Baibing Li, Xiangkang Yin, and Jing Zhao. 2019. "Variables in Dollar Terms Versus in Rate Terms: The Case of Market Feedback on Merger Negotiations". figshare. https://hdl.handle.net/2134/24396. 


\title{
Variables in Dollar Terms versus in Rate Terms: The Case of Market Feedback on Merger Negotiations
}

\author{
Hsin-I Chou ${ }^{\mathrm{a}}$, Baibing Li ${ }^{\mathrm{b}}$, Xiangkang Yin ${ }^{\mathrm{c}, *}$, and Jing Zhao ${ }^{\mathrm{c}}$
}

${ }^{a}$ Department of Accountancy and Finance, University of Otago, New Zealand.

${ }^{\mathrm{b}}$ School of Business and Economics, Loughborough University, United Kingdom.

${ }^{\mathrm{c}}$ Department of Economics and Finance, La Trobe Business School, La Trobe University, Australia.

* Corresponding author, Department of Economics and Finance, La Trobe Business School, La Trobe University, Bundoora, VIC 3086, Australia. Tel: +61 39479 2312, Email: x.yin@latrobe.edu.au. The authors are grateful to Yangyang Chen (discussant), the referee and seminar participants at La Trobe University and the $23^{\text {rd }}$ Conference on the Theories and Practices of Securities and Financial Markets for their highly valuable comments. Xiangkang Yin and Jing Zhao acknowledge the Discovery Projects funding from Australian Research Council (DP140100113).

[Type text] 


\title{
Variables in Dollar Terms versus in Rate Terms: The Case of Market Feedback on Merger Negotiations
}

\begin{abstract}
This paper shows a sharp contrast between theoretical predictions of merger negotiations when takeover markup and runup are measured in dollar vs rate terms. It argues that the empirical tests by an influential study cannot reject the hypothesis of a costly feedback loop as the authors claim. Using markup and runup in standardized dollar terms, it provides evidence that is consistent with both hypotheses of rational deal anticipation and a costly feedback loop. This exercise demonstrates the importance and necessity of differentiating regressions with variables in dollar terms and in rate terms to avoid drawing inaccurate or even false conclusions.
\end{abstract}

JEL Classification: G34

Keywords: Takeover, offer premium, runup, markup, feedback loop

[Type text] 


\section{Introduction}

How do takeover bidders react to the market feedback on merger negotiations? Do they have to pay higher takeover costs if the target stock prices experience substantial pre-offer runups? In a seminal study, Schwert (1996) reports that in a large sample of takeovers, bidding firms markup their offers almost equal to the runups. This finding implies that markup pricing prevails in the competition for corporate control (see also Betton, Eckbo and Thorburn (2008)). Recently, Betton, Eckbo, Thompson and Thorburn (2014, BETT hereafter) develop an empirically testable model of takeover that permits stock market feedback on takeover rumors. ${ }^{1}$ The model assumes that the information of takeover negotiations is leaked in the form of rumors which send a signal to the market of the ongoing negotiations. An important insight generated by the model is that takeover rumors or signals reveal the information of "both the deal probability and the dealspecific takeover synergies conditional on a bid.” Rational investors use the signal "to update not only the takeover probability but also the conditional value" of synergies. With this endogenous deal probability, the bidders have different offering strategies depending on whether the market is operating under rational deal anticipation or there is a costly feedback loop in the takeover negotiations. The model predicts that in a linear regression of takeover markup on runup, the slope coefficient is greater than -1 under rational deal anticipation. However, the coefficient is strictly positive if there is a costly feedback loop from takeover runup to markup. BETT conducts a thorough and solid empirical analysis to test the two competing hypotheses.

\footnotetext{
${ }^{1}$ Research on mergers and acquisitions is extensive. For a general survey, see Betton, Eckbo and Thorburn (2008); for rumors on mergers and acquisitions and their effects on pricing, see, for example, Jarrell and Poulsen (1989), Pound and Zeckhauser (1990), and Zivney, Bertin and Torabzadeh (1996).
}

[Type text] 
Based on the negative slope coefficients of their linear regressions, the authors claim that their empirical results support the hypothesis of rational deal anticipation and reject that of a costly feedback loop.

This paper is concerned by the lack of connection between the theory and the empirical tests in BETT. The theoretical predictions made by the takeover model are based on markup and runup in terms of dollar values, whereas the various empirical tests conducted by BETT use the rates of markup and runup. We argue that the relationship between markup and runup in dollar terms is different from that between the rates of markup and runup. To this end, we establish, under the BETT framework, that the slope coefficient of a linear regression of the markup rate against the runup rate can be negative under both the hypotheses of rational deal anticipation and a costly feedback loop. Thus, in contradiction to their claim, BETT's empirical results do not reject either hypothesis.

To see the point intuitively, let us consider a numerical example. Following BETT's notation, $V_{R}$ is the runup of the target firm's value after the market receives a signal of the potential takeover and $V_{P}$ is the market's valuation of the target's deal value conditional on the bid announcement (i.e., the takeover premium). Both $V_{R}$ and $V_{P}$ are in dollar terms. Assuming that the initial value of the target is $V_{I},{ }^{2}$ then the target's market value after stock price run-up but just before takeover announcement is $V_{I}+V_{R}$ and its value after takeover announcement is $V_{I}+V_{P}$. In turn, the target's markup in dollar terms is $V_{I}+V_{P}-\left(V_{I}+V_{R}\right)=V_{P}-V_{R}$, and the rates of runup and markup can be defined by $R_{R}=\frac{V_{R}}{V_{I}}$ and $R_{M}=\frac{V_{P}-V_{R}}{V_{I}+V_{R}}$. BETT empirically examines various forms of the following regression model,

\footnotetext{
${ }^{2}$ It is the value before the market receives the takeover signal. BETT normalizes it to zero in the theoretical model.
} 


$$
R_{M}=\tilde{a}+\tilde{b} R_{R},
$$

and the results are reported in their Table IV. Here we use $\tilde{a}=0.36$ and $\tilde{b}=-0.24$ from model (1) in Table IV of BETT to generate a dataset of $R_{M}$ and $R_{R}$, which is illustrated in Panel A of Figure 1 , where $R_{R}$ is exogenous, ranging from 0 to $30 \%$. Since $V_{R}=V_{I} R_{R}$ and $V_{P}-V_{R}=$ $V_{I}\left(1+R_{R}\right) R_{M}$, we obtain dollar-value runups and markups in Panel $\mathrm{B}$ that correspond to the rates of runups and markups in Panel A. Figure 1 shows that although the dataset presents a negative correlation between the rates of runup and markup, the corresponding correlation between dollar-value runup and markup is actually positive. In other words, a negative estimate of $\tilde{b}$ in regression (1) does not secure a negative slope $b$ in the regression of dollar-value markup, $V_{P}-V_{R}$, against dollar-value runup, $V_{R}$; i.e.,

$$
V_{P}-V_{R}=a+b V_{R} .
$$

Therefore, the empirical results reported in Table IV of BETT do not contradict the hypothesis of rational deal anticipation, nor do they provide evidence rejecting the hypothesis of a costly feedback loop.

\section{Figure 1 here}

Figure 1 has more general implications. Researchers often analyze price (or value) changes when developing theories or modeling to preserve tractability, and they analyze the rates of returns when empirically testing the theories to preserve the comparability in the cross-section (see, for example, Chordia and Subrahmanyam (2004), Hong and Stein (1999), and Hong, Lim and Stein (2000)). In some cases, this distinction has no material implications for the understanding of economic forces and mechanisms. However, the negative (or positive) relationship implied by the linear regression of the rates of two random economic variables may not be retained for their dollar-value counterparts. Therefore, further examination and validation 
are required when the estimates from the regressions of the rates are used to test a hypothesis based on the predictions for the variables in their dollar terms. A leading example in this regard is Banerjee (2011), who shows the difference between dollar return and the rate of return by theoretically analyzing the effects of dispersion in beliefs on dollar returns separately from the effects on the rates of return.

The remainder of this paper is organized as follows. Section 2 briefly reviews the BETT model and its main findings. Section 3 presents the theoretical predictions on the relationship between the rates of runup and markup in the BETT framework. Under rational deal anticipation, they are, to a certain extent, consistent with the predictions in Proposition 1 of BETT. However, the predictions exhibit substantial differences under the hypothesis of a costly feedback loop. The key finding is that under both hypotheses, the relationship between the markup rate and runup rate can be either positive or negative, depending on the model parameters. Therefore, the sign (or the range) of the slope coefficient of linear regression (1) cannot test these hypotheses.

To empirically examine the hypotheses of rational deal anticipation and a costly feedback loop, Section 4 proposes using standardized runup and markup to test the two hypotheses; i.e., both dollar-value runup and markup are scaled by the initial value of the target. The empirical results indicate that the hypothesis of a costly feedback loop cannot be rejected and rational deal anticipation should not be considered a favored hypothesis. This claim contradicts BETT's conclusion. The final section concludes the paper. 


\section{A Review of the BETT Model}

The market receives a rumor (takeover signal), $s$, after the negotiations of a takeover start, and the signal causes investors to anticipate a synergistic takeover. The value of total synergies for the takeover is $S$, which is known to the bidder and target negotiators but is unknown to the market. However, the market knows the distribution of $S$ upon the reception of signal $s$, i.e., conditional probability density function $g(S \mid s)$ and cumulative distribution function $G(S \mid s)$ are public knowledge. The rule of synergy sharing is that the acquirer receives $\theta S$, while the target receives $B(S) \equiv(1-\theta) S$.

The baseline takeover model assumes rational deal anticipation; that is, the takeover offer price does not respond to the takeover runup before the offer announcement. As the bidder bears a known bidding cost $C$, the bid only occurs if $S>K \equiv C / \theta$. Therefore, the probability of the bid occurring can be calculated by

$$
\pi=\int_{K}^{\infty} g(S \mid s) d S
$$

The offer premium, conditional on the offer announcement, is $V_{P}=\bar{B} \equiv E(B(S) \mid s, b i d)$. In turn, the runup in dollar terms, $V_{R}$, has the following relationship with $V_{P}$ :

$$
V_{R}=\int_{K}^{\infty} B(S) g(S \mid s) d S=\pi V_{P} .
$$

Therefore, the markup and runup are related through

$$
V_{P}-V_{R}=\frac{1-\pi}{\pi} V_{R}
$$

PROPOSITION 1 OF BETT: Suppose the markup projection (3) holds. When the takeover signal causes the market to infer different takeover probabilities and conditional deal values 
across a sample of takeovers ( $d \pi / d s>0$ and $d V_{P} / d s>0$ ), then the linear regression (2) produces a slope coefficient $b$ that is strictly greater than -1 .

The costly feedback loop implies that there is a runup transfer $V_{R}^{*}$ to the target in addition to the announcement surprise $\bar{B}^{*} \equiv E^{*}(B(S) \mid s, b i d)$. Therefore, the takeover premium has two components:

$$
V_{P}^{*}=\bar{B}^{*}+V_{R}^{*}
$$

The superscript * indicates values and expectations computed using the new bid threshold $K^{*}=\left(C+V_{R}^{*}\right) / \theta$. Due to the increased bid threshold, the probability of the bid occurring now becomes

$$
\pi^{*}=\int_{K^{*}}^{\infty} g(S \mid s) d S
$$

With this probability, we still have $V_{R}^{*}=\pi^{*} V_{P}^{*}$ and

$$
V_{P}^{*}-V_{R}^{*}=\bar{B}^{*}=\frac{1-\pi^{*}}{\pi^{*}} V_{R}^{*} .
$$

PROPOSITION 2 OF BETT: Suppose the markup projection (4) holds. When merger negotiations force rational bidders to raise the offer price with the runup (costly feedback loop), the markup becomes a positive and monotonic function of the runup, and the linear markup regression (2) yields a positive slope coefficient $(b>0)$.

\section{The Relationship between the Rates of Runup and Markup}

BETT's theoretical model of takeover and its predictions are developed in terms of dollar values, whereas the corresponding empirical analysis is based on the rates of runup and markup. 
To examine the implications and relevance of their empirical analysis, we need a theory and predictions based on markup and runup rates.

\subsection{The Baseline Model}

This subsection presents the rate-version of theoretical predictions about the effects of runup on markup, under the rational deal anticipation. Corresponding to Proposition 1 of BETT, we first demonstrate the following proposition.

PROPOSITION 1: Suppose the markup projection (3) holds and the takeover signal causes the market to infer different takeover probabilities and conditional deal values (d $\pi / d s>0$ and $\left.d V_{P} / d s>0\right)$. Then, the change in markup rate with respect to the change in runup rate, $d R_{M} / d R_{R}$, is

(i) strictly greater than -1 if the takeover probability is greater than or equal to $50 \%$ $\left(\pi \geq \frac{1}{2}\right)$

(ii) strictly greater than $\frac{-1}{4 \pi(1-\pi)}$ if the takeover probability is smaller than $50 \%\left(\pi<\frac{1}{2}\right)$;

(iii) strictly smaller than $\frac{(1-\pi) V_{I}^{2}}{\pi\left(V_{I}+V_{R}\right)^{2}}<\frac{1-\pi}{\pi}$.

Proof: See Appendix A.

Proposition 1 implies that the slope of linear regression (1) for the rate of markup against the rate of runup has a lower bound of -1 only if all takeover bids are more likely to occur (i.e., probability $\pi \geq 1 / 2$ ). This result is parallel to Proposition 1 of BETT for the regression of $V_{P}-V_{R}=a+b V_{R}$. BETT's empirical analysis was undertaken for the rates of markup and 
runup (see Tables III and IV in BETT), hence their empirical results are consistent with Proposition 1. However, because $\frac{-1}{4 \pi(1-\pi)}<-1$, Proposition 1 also implies that the slope of linear regression $R_{M}=\tilde{a}+\tilde{b} R_{R}$ can be less than -1 if there is a substantial portion of observations with $\pi<1 / 2$ in the sample. The regression results reported in Table IV of BETT show that $\tilde{b}>-1,{ }^{3}$ which implies that the market on average expects that the takeover deal is more likely to occur if the hypothesis of rational deal anticipation is true.

Result (iii) in Proposition 1 imposes an upper bound for the derivative $d R_{M} / d R_{R}$. At the extreme, if the initial value of the target $V_{I}$ is very small relative to its value after runup, $V_{I}+V_{R}$, and/or if the market considers the takeover bid extremely likely to occur (i.e., $\pi$ is close to 1 ), then the upper bound $\frac{(1-\pi) V_{I}^{2}}{\pi\left(V_{I}+V_{R}\right)^{2}}$ approaches zero (see Figure 2 below for illustration). In other words, the slope coefficient $\tilde{b}$ of regression (1) is most likely to be negative in this case.

Throughout the paper, BETT uses the example of uniformly distributed synergy $S$ extensively to illustrate the insights and implications of the theoretical model. Following BETT, we consider $G(S \mid s) \sim U(s-\Delta, s+\Delta)$ and $g(S \mid s)=1 / 2 \Delta$. Applying the values of $\pi, V_{R}, V_{P}, \frac{d V_{R}}{d s}$ and $\frac{d V_{P}}{d s}$ given in BETT's Appendix, we can easily show that when $s-\Delta<K<s+\Delta$, there is

$$
\frac{d R_{M}}{d R_{R}}=-\frac{4 V_{I} s+(1-\theta)(s+\Delta+K)^{2}}{4\left(V_{I}+V_{R}\right)^{2}(s+\Delta)} V_{I}
$$

This leads us to the following corollary.

\footnotetext{
${ }^{3}$ The only exception is model (2), which returns an estimate $\tilde{b}$ equal to -1.01 . Although the estimate of -1.01 is insignificantly different from -1, BETT takes it as potential violation to their theoretical predictions and attributes the excess of -1 as the result of runup adjustment.
}

[Type text] 
COROLLARY 1: Suppose the markup projection (3) holds and synergy $S$ is uniformly distributed on $(s-\Delta, s+\Delta)$. If the bidding cost is in a reasonable range that $s-\Delta<K<s+$ $\Delta$, then $\frac{d R_{M}}{d R_{R}}$ satisfies (5).

Proof: See Appendix B.

Obviously, the corollary indicates that the slope of linear regression $R_{M}=\tilde{a}+\tilde{b} R_{R}$ is negative if all of the takeover signals are positive. This property of $\tilde{b}$ is qualitatively similar to the slope of $b$ in the regression of dollar-value markup and runup. However, (5) predicts that the relationship between the rate of markup and the rate of runup depends on the size of the target's stand-alone value. For example, $\frac{d R_{M}}{d R_{R}}$ tends to zero when $V_{I}$ tends to zero.

Panel A of Figure 2 illustrates derivative $\frac{d R_{M}}{d R_{R}}$ against the increase in the takeover signal $s$ received by the market, as specified by (5). Apparently, the relationship between $R_{M}$ and $R_{R}$ is highly nonlinear. The derivative declines sharply in the negative territory of $s$ and reaches a minimum below zero. Then, it gradually increases and moves toward zero. Intuitively, when the signal is very low and negative, the market does not really expect that the potential bidding firm will eventually offer to buy the target (i.e., $\pi$ is close to zero). Therefore, a marginal improvement in signal $s$ triggers a moderate runup but a profound announcement surprise if the takeover offer is eventually announced. This effect is reflected by the high upper bound $\frac{1-\pi}{\pi}$ and the large derivative $\frac{d R_{M}}{d R_{R}}$. With the rise of signal $s$, the market expectation of takeover probability increases substantially and the marginal effect of signal on runup rate is intensified, but the effect on announcement surprise is weakened, which leads $\frac{d R_{M}}{d R_{R}}$ to fall steeply. At $s=-0.048, \frac{d R_{M}}{d R_{R}}$ 
reaches its minimum of -0.342 . After this minimum point, a stronger signal has a greater effect on the market interpretation of conditional deal value. Therefore, a large signal induces a greater markup rate than runup rate, resulting in an increasing $\frac{d R_{M}}{d R_{R}}$.

\section{Figure 2 here}

\subsection{The Markup Accommodating a Costly Feedback Loop}

With a costly feedback loop, the rate of markup is

$$
R_{M}^{*}=\frac{V_{P}^{*}-V_{R}^{*}}{V_{I}+V_{R}^{*}}=\frac{\bar{B}^{*}}{V_{I}+V_{R}^{* \prime}}
$$

and

$$
\frac{d R_{M}^{*}}{d R_{R}^{*}}=\frac{V_{I}}{\left(V_{I}+V_{R}^{*}\right)^{2}}\left\{\left(\frac{d \bar{B}^{*}}{d s} / \frac{d V_{R}^{*}}{d s}\right)\left(V_{I}+V_{R}^{*}\right)-\bar{B}^{*}\right\} .
$$

However, reorganizing Eq (15) in BETT yields

$$
\frac{d \bar{B}^{*}}{d s} / \frac{d V_{R}^{*}}{d s}=\frac{1-\pi^{*}}{\pi^{*}}\left(1+\frac{\bar{B}^{*} g\left(K^{*}\right)}{\theta\left(1-\pi^{*}\right)^{2}}-\frac{\bar{B}^{*} \Upsilon}{\left(1-\pi^{*}\right)^{2}}\right)
$$

where $\Upsilon \equiv\left(\frac{d V_{R}^{*}}{d s}\right)^{-1} \int_{K^{*}}^{\infty} g^{\prime}(S \mid s) d S$. Substituting the above equation into (6) and noting $\bar{B}^{*}=$ $\frac{1-\pi^{*}}{\pi^{*}} V_{R}^{*}$, we have

$$
\frac{d R_{M}^{*}}{d R_{R}^{*}}=\frac{V_{I}\left(1-\pi^{*}\right)}{\left(V_{I}+V_{R}^{*}\right)^{2} \pi^{*}}\left[\left(V_{I}+V_{R}^{*}\right)\left(1+\frac{V_{R}^{*} g\left(K^{*}\right)}{\theta \pi^{*}\left(1-\pi^{*}\right)}-\frac{V_{R}^{*} \Upsilon}{\pi^{*}\left(1-\pi^{*}\right)}\right)-V_{R}^{*}\right] .
$$

Hence, $\frac{d R_{M}^{*}}{d R_{R}^{*}}<0$ if and only if

$$
\frac{V_{R}^{*}}{\pi^{*}\left(1-\pi^{*}\right)}\left(\Upsilon-\frac{g\left(K^{*}\right)}{\theta}\right)>\frac{V_{I}}{V_{I}+V_{R}^{*}} .
$$


Since the right side of (7) is positive, a necessary condition for (7) to be true is that $\Upsilon>$ $g\left(K^{*}\right) / \theta$. Noting that $\Upsilon$ is inversely related to $\frac{d V_{R}^{*}}{d s}$, we find that the inequality is more likely to hold if dollar-value runup is less sensitive to takeover rumor. Given $\Upsilon>g\left(K^{*}\right) / \theta$, we can see from (7) that it is more likely to hold if the stand-alone value of the target is relatively smaller than the runup. In general, the model cannot rule out the possibility of the relationship between the rates of markup and runup being positive or negative without further specifications. This is in sharp contrast to the monotonic and positive relationship between markup and runup in dollar terms, as predicted by Proposition 2 of BETT.

PROPOSITION 2: Suppose the markup projection (4) holds. When merger negotiations force rational bidders to raise the offer price with the runup (costly feedback loop), the rate of markup can be either positively or negatively related to the rate of runup, and the slope coefficient in the linear regression $R_{M}^{*}=\tilde{a}+\tilde{b} R_{R}^{*}$ can be either positive or negative.

Propositions 1 and 2 have an important implication for empirical analysis. They state that the slope coefficient in the linear regression of the rate of markup over the rate of runup can be either positive or negative under both hypotheses of rational deal anticipation and a costly feedback loop. Therefore, it is impossible to use the sign of the slope coefficient of (1) to support or reject either hypothesis.

To have a closer look on condition (7), we now consider again that synergy $S$ is uniformly distributed, i.e., $G(S \mid s) \sim U(s-\Delta, s+\Delta)$. Since $\pi^{*}=1$ if $K^{*}<s-\Delta$ and $\pi^{*}=0$ if $K^{*}>s+\Delta$, we assume a market with $K^{*}-\Delta<s<K^{*}+\Delta$ to avoid the trivial cases. With this 
specification, we can obtain closed-form solutions to all of the variables and, in turn, attain the following corollary.

COROLLARY 2: Suppose the markup projection (4) holds and takeover synergy S is uniformly distributed on $(s-\Delta, s+\Delta)$. When a costly feedback loop prevails in the takeover market,

(i) the derivative $\frac{d R_{M}^{*}}{d R_{R}^{*}}$ is negative if and only if

$$
\frac{4 \Delta^{2} \theta^{2} V_{R}^{*}\left[V_{R}^{*}+2 \Delta \theta^{2}+C-(s+\Delta) \theta^{2}\right]}{\left[(s+\Delta) \theta-C-V_{R}^{*}\right]\left[(\Delta-s) \theta+C+V_{R}^{*}\right]\left[V_{R}^{*}+(1-\theta)(s+\Delta)\right]}>\frac{V_{I}}{V_{I}+V_{R}^{*}}
$$

where $V_{R}^{*}$ is determined by

$$
V_{R}^{*}=\frac{\sqrt{\left[(\Delta-s) \theta^{2}+C\right]^{2}+\left(1-\theta^{2}\right)\left[(s+\Delta)^{2} \theta^{2}-C^{2}\right]}-\left[(\Delta-s) \theta^{2}+C\right]}{(1+\theta)} ;
$$

(ii) the derivative $\frac{d R_{M}^{*}}{d R_{R}^{*}}$ is strictly greater than $\frac{\pi^{*}-1}{4 \pi^{*}}$ but smaller than $\frac{V_{I}^{2}}{\left(V_{I}+V_{R}^{*}\right)^{2}} \frac{1-\pi^{*}}{\pi^{*}}<\frac{1-\pi^{*}}{\pi^{*}}$.

Proof: See Appendix C.

The corollary confirms the results in Proposition 2 that the takeover model of BETT does not rule out the possibility that the slope coefficient of linear regression $R_{M}^{*}=\tilde{a}+\tilde{b} R_{R}^{*}$ could be either positive or negative under the hypothesis of a costly feedback loop. Panel B in Figure 2 illustrates this possibility and it is obvious that the curve of $\frac{d R_{M}^{*}}{d R_{R}^{*}}$ in the panel is stunningly similar to the curve of $\frac{d R_{M}}{d R_{R}}$ in Panel A of Figure 2. This similarity between the two paradigms implies that it is extremely difficult to separate the two hypotheses by the slope coefficient of the linear regression of the rate of markup on the rate of runup.

It is interesting to note that the upper bound for the case of a costly feedback loop in Corollary 2 has the same functional form as that of the baseline model in Proposition 1. [Type text] 
Moreover, although the lower bound $\frac{\pi^{*}-1}{4 \pi^{*}}$ in Corollary 2 requires the assumption of a uniform distribution of synergy $S$, the upper bound of $\frac{V_{I}^{2}}{\left(V_{I}+V_{R}^{*}\right)^{2}} \frac{1-\pi^{*}}{\pi^{*}}$ does not rely on this assumption. As the proof of Corollary 2 shows, it holds as long as the probability of a bid occurring $\pi^{*}$ increases in signal $s .4$

\section{Can the Hypothesis of a Costly Feedback Loop Be Rejected?}

As we have seen, the theoretical model developed in Section 3 does not directly present unambiguous predictions of the relationship between the rates of runup and markup for the empirical tests of rational deal anticipation vs. a costly feedback loop in the takeover markets. However, the model's predictions based on dollar-value runup and markup are less ambiguous. The problem of regressing dollar-value markup against dollar-value runup in cross-sectional analysis is that markup and runup vary substantially in cross-section, which may invalidate the regression. To overcome this difficulty, we propose using standardized runup and markup; that is, each target firm's runup and markup are scaled by its initial value. More specifically, we define standardized runup and takeover premium as $W_{R} \equiv \frac{V_{R}}{V_{I}}$ and $W_{P} \equiv \frac{V_{P}}{V_{I}}$. Thus, the standardized markup is $W_{P}-W_{R}$. As the initial value $V_{I}$ is independent of the takeover signal $s$, we have $\frac{d\left(V_{P}-V_{R}\right)}{d s}=\frac{V_{I} d\left(W_{P}-W_{R}\right)}{d s}$ and $\frac{d V_{R}}{d s}=\frac{V_{I} d W_{R}}{d s}$. Therefore, $\frac{d\left(V_{P}-V_{R}\right)}{d V_{R}}=\frac{d\left(W_{P}-W_{R}\right)}{d W_{R}}$ and BETT's Propositions 1 and 2 hold for the standardized runup and markup. This section uses (2a) below to undertake empirical analysis and investigate further whether the hypothesis of a costly feedback loop can be rejected:

\footnotetext{
${ }^{4}$ The assumption of uniformly distributed $S$ conditional on signal $s$ ensures that $\pi^{*}$ increases in $s$.
} 


$$
W_{P}-W_{R}=a+b W_{R}
$$

To facilitate the comparison of our results with those of BETT, we adopt the sample selection criteria set by BETT to select the sample. The initial control bids are collected from SDC Platinum of Thomson Reuters with the following criteria: the transaction form should be "merger" or "acquisition of majority interest", the target company must be publicly listed and U.S. domiciled, and the acquirer company should own less than $50 \%$ of the target shares prior to the merger and acquisition and seek to own at least $50 \%$ of the target shares. Following BETT, we further require that the targets should be listed on NYSE, Amex or NASDAQ; have at least 100 days of common stock return data from CRSP over the estimation period; have a total market capitalization exceeding $\$ 10$ million on day $-42 ;{ }^{5}$ have a stock price greater than $\$ 1$ on day -42 ; have an offer price in the SDC database; have stock price information in CRSP on day -2 ; have an announcement return for the window $[-1,+1]$; have information on the outcome and ending date of the contest; and have a contest length of less than or equal to 252 trading days (one year). Similar to BETT, we drop the subsequent control bids within six months of an earliest control bid. BETT's sample period is from January 1980 to December 2008, and we extend it to December 2013. To ensure that our empirical findings are not sensitive to the sample period selection, the results from both sample periods are reported. The final sample has 6953 control contests. Table I reports the annual distributions of the rate of offer premium, the rate of markup, the rate of runup (which is equal to the standardized runup) and the standardized markup. The standard deviations of these variables are also reported at the bottom of the table.

$$
\text { Table I here }
$$

\footnotetext{
${ }^{5}$ Event day, i.e., takeover offer announcement day, is defined as day zero.
} 
We use three sets of variables to proxy scaled dollar-value markup and runup. First, we set $W_{P}-W_{R}=\frac{O P-P_{-2}}{P_{-42}}$ and $W_{R}=\frac{P_{-2}}{P_{-42}}-1$, where $O P$ is the price per share offered by the initial bidder and $P_{t}$ is the target's stock price on day $t$ relative to the takeover announcement day $(t=0)$. In the second set, we consider takeover markup over three days around takeover announcement, and therefore $W_{P}-W_{R}=\frac{P_{1}-P_{-2}}{P_{-42}}$ and $W_{R}=\frac{P_{-2}}{P_{-42}}-1$.

In these two sets of variables, the effect of systematic risk has not been eliminated. To adjust for systematic risk, let us consider the rate of abnormal return $r_{t}=\frac{P_{t}-P_{t-1}}{P_{t-1}}-\beta m_{t}$ on day $t$, where $\beta$ is the market beta of the target firm and $m_{t}$ is market return on day $t$. If there are $N$ shares outstanding, the abnormal increase of the target value on day $t$, in dollar terms, is $a v_{t}=N P_{t-1} r_{t}=N\left(P_{t}-P_{t-1}-\beta m_{t} P_{t-1}\right)$. Since the abnormal increase in a period is the sum of $a v_{t}$ over the period, the adjusted-runup can be defined as the abnormal increase of the target value in the runup period adjusted by systematic risk, i.e., $A V_{R}=N\left(P_{-2}-P_{-42}\right)-$ $N \beta \sum_{t=-41}^{t=-2} m_{t} P_{t-1}$. Similarly, the adjusted-markup is $A V_{M}=N\left(P_{1}-P_{-2}\right)-N \beta \sum_{t=-1}^{t=1} m_{t} P_{t-1}$. Scaling them by the target's value on the initial day, $N P_{-42}$, we have the standardized adjustedrunup

$$
S A V_{R}=\frac{P_{-2}-P_{-42}}{P_{-42}}-\frac{\beta}{P_{-42}} \sum_{t=-41}^{t=-2} m_{t} P_{t-1}
$$

and the standardized adjusted-markup

$$
S A V_{M}=\frac{P_{1}-P_{-2}}{P_{-42}}-\frac{\beta}{P_{-42}} \sum_{t=-1}^{t=1} m_{t} P_{t-1} .
$$

Therefore, the third set of variables are $W_{P}-W_{R}=S A V_{M}$ and $W_{R}=S A V_{R}$. 
The regression outcomes of these three sets of variables are documented in the left panel of Table II, in which a regression analysis of (2a) is performed based on each set of variables for both 1980-2008 and 1980-2013 sample periods. Obviously, all of the slope coefficients are positive and statistically significant. ${ }^{6}$ Because supporting the hypothesis of rational deal anticipation and at the same time rejecting that of a costly feedback loop require a significant slope coefficient between minus one and zero, the empirical results in the left panel of Table II do not provide evidence in favor of rational deal anticipation in the market for corporate control. On the other hand, the right panel of Table II shows that the slope coefficients of linear regression (1) of the rate of markup on the rate of runup are between minus one and zero and significant, which are consistent with the results reported in Table IV of BETT for similar regressions. This sharp contrast of the slope coefficients between the pairs of regressions in the left and right panels of Table II demonstrates that extra caution should be exerted when applying outcomes from the regression model of the rates of variables to the analysis of theoretical predications based on their counterparts in dollar-terms.

\section{Table II here}

\section{Concluding Remarks}

In contrast to BETT's claim, this paper provides theoretical and empirical evidence showing that both hypotheses of rational deal anticipation and a costly feedback loop cannot be rejected. On the theoretical front, we demonstrate that the relationship between the rates of markup and runup can be negative under both hypotheses, which contradicts the theoretical

\footnotetext{
${ }^{6}$ Noting that the residuals in (2a) are likely to be heteroscedastic, we also use heteroscedasticity-consistent tstatistics to examine the significance of the slope coefficient and the results are qualitatively indifferent.
}

[Type text] 
prediction of the monotonic and positive relationship between dollar-value markup and runup under a costly feedback loop hypothesis. On the empirical analysis side, we document that the slope coefficient of the linear regression of standardized markup and runup is (significantly) positive, which is consistent with both hypotheses of rational deal anticipation and a costly feedback loop.

More importantly, this paper advocates the importance and necessity of scrutinizing the implications of the regression of variables in dollar terms and that of these variables in their rates, if theory explores the economic mechanisms using dollar-value variables to preserve tractability and an empirical analysis is conducted by the rates of these variables to facilitate cross-sectional comparison. Without thorough scrutiny, it is likely to run the risk of drawing inaccurate or even incorrect conclusions when using empirical results to support or reject the theoretical predictions.

[Type text] 


\section{Appendix: Proofs}

\section{A. Proof of Proposition 1}

Taking derivative of $R_{M}=\frac{V_{P}-V_{R}}{V_{I}+V_{R}}$ with respective to $s$ and applying (3) yield

$$
\frac{d R_{M}}{d s}=\frac{\pi\left(V_{I}+V_{R}\right) \frac{d V_{P}}{d s}-\left(\pi V_{I}+V_{R}\right) \frac{d V_{R}}{d s}}{\pi\left(V_{I}+V_{R}\right)^{2}} .
$$

Substituting $\frac{d V_{P}}{d s}=\frac{1}{\pi^{2}}\left(\pi \frac{d V_{R}}{d s}-V_{R} \frac{d \pi}{d s}\right)$ into it leads to

$$
\frac{d R_{M}}{d s}=\frac{\left(V_{I}+V_{R}\right)\left(\pi \frac{d V_{R}}{d s}-V_{R} \frac{d \pi}{d s}\right)-\pi\left(\pi V_{I}+V_{R}\right) \frac{d V_{R}}{d s}}{\pi^{2}\left(V_{I}+V_{R}\right)^{2}} .
$$

Noting $\frac{d V_{R}}{d s}>0$, we have $\frac{d R_{R}}{d s}=\frac{1}{V_{I}} \frac{d V_{R}}{d s}>0$. Therefore,

$$
\frac{d R_{M}}{d R_{R}}<\frac{(1-\pi) V_{I}^{2}}{\pi\left(V_{I}+V_{R}\right)^{2}}<\frac{1-\pi}{\pi}
$$

which proves conclusion (iii) of the proposition. On the other hand, dropping the term related to $\frac{d V_{P}}{d s}$ in (A1) results in

$$
\frac{d R_{M}}{d R_{R}}>-\frac{V_{I}\left(\pi V_{I}+V_{R}\right)}{\pi\left(V_{I}+V_{R}\right)^{2}} .
$$

It is easy to verify that when $\pi \geq 1 / 2, \frac{V_{I}\left(\pi V_{I}+V_{R}\right)}{\pi\left(V_{I}+V_{R}\right)^{2}}$ is a strictly decreasing function of $V_{R}$ for any $V_{R} \geq 0$, and hence it attains the maximum of 1 at $V_{R}=0$. If $\pi<1 / 2, \frac{V_{I}\left(\pi V_{I}+V_{R}\right)}{\pi\left(V_{I}+V_{R}\right)^{2}}$ attains the unique global maximum of $\frac{1}{4 \pi(1-\pi)}$ at $V_{R}=(1-2 \pi) V_{I}$. This completes the proof. 


\section{B. Proof of Corollary 1}

With uniformly distributed $S$ and non-triviality condition $s-\Delta<K<s+\Delta$, BETT demonstrates $\quad$ that $\quad \pi=\frac{s+\Delta-K}{2 \Delta}, \quad V_{R}=\frac{1-\theta}{4 \Delta}\left[(s+\Delta)^{2}-K^{2}\right], \quad V_{P}=\frac{1-\theta}{2}(s+\Delta+K), \quad \frac{d V_{R}}{d s}=$ $\frac{1-\theta}{2 \Delta}(s+\Delta)$ and $\frac{d V_{P}}{d s}=\frac{1-\theta}{2}$. Applying them to $\frac{d R_{M}}{d R_{R}}$, we obtain

$$
\begin{aligned}
\frac{d R_{M}}{d R_{R}} & =\frac{\Delta\left(V_{I}+\frac{1-\theta}{4 \Delta}\left[(s+\Delta)^{2}-K^{2}\right]\right)-\left(V_{I}+\frac{1-\theta}{2}(s+\Delta+K)\right)(s+\Delta)}{\left(V_{I}+V_{R}\right)^{2}(s+\Delta)} V_{I} \\
& =-\frac{4 V_{I} s+(1-\theta)(s+\Delta+K)^{2}}{4\left(V_{I}+V_{R}\right)^{2}(s+\Delta)} V_{I} .
\end{aligned}
$$

\section{Proof of Corollary 2}

From the definitions, we have

$$
\pi^{*}=\frac{(s+\Delta) \theta-C-V_{R}^{*}}{2 \Delta \theta}, \quad \bar{B}^{*}=\frac{1-\theta}{2 \theta}\left[(s+\Delta) \theta+C+V_{R}^{*}\right] .
$$

Since $1-\pi^{*}=\frac{(\Delta-s) \theta+C+V_{R}^{*}}{2 \Delta \theta}$ and $\bar{B}^{*}=\frac{1-\pi^{*}}{\pi^{*}} V_{R}^{*}$, there is

$$
\frac{1-\theta}{2 \theta}\left[(s+\Delta) \theta+C+V_{R}^{*}\right]=\frac{(\Delta-s) \theta+C+V_{R}^{*}}{(s+\Delta) \theta-C-V_{R}^{*}} V_{R}^{*}
$$

Reorganizing it yields

$$
(1+\theta)\left(V_{R}^{*}\right)^{2}+2\left[(\Delta-s) \theta^{2}+C\right] V_{R}^{*}-(1-\theta)\left[(s+\Delta)^{2} \theta^{2}-C^{2}\right]=0 .
$$

The solution to (A2) is $V_{R}^{*}$ in (9), where we have dropped the solution that leads to $V_{R}^{*}<0$. On the other hand, taking the derivative of (A2) with respect to $s$ yields

$$
\frac{d V_{R}^{*}}{d s}=\frac{\theta^{2} V_{R}^{*}+(1-\theta)(s+\Delta) \theta^{2}}{(1+\theta) V_{R}^{*}+(\Delta-s) \theta^{2}+C}>0 .
$$

For the uniform distribution on $(s-\Delta, s+\Delta), g\left(K^{*}\right)=1 /(2 \Delta)$ and $\int_{K^{*}}^{\infty} g^{\prime}(S \mid s) d S=1 /(2 \Delta)$. Applying them and $\frac{d V_{R}^{*}}{d s}$ in (A3) to solve for $\Upsilon$ in (7), we obtain (8) in the text. 
For the lower bound in (ii), first noting that $\bar{B}^{*}=\frac{1-\theta}{2 \theta}\left[(s+\Delta) \theta+C+V_{R}^{*}\right]$, we have

$$
\frac{d \bar{B}^{*}}{d s}=\frac{1-\theta}{2 \theta}\left(\theta+\frac{d V_{R}^{*}}{d s}\right)>\frac{1-\theta}{2}
$$

Applying it to (6) yields

$$
\frac{d R_{M}^{*}}{d R_{R}^{*}}>\frac{V_{I}}{\left(V_{I}+V_{R}^{*}\right)^{2}}\left\{\frac{1-\theta}{2 \theta}\left(V_{I}+V_{R}^{*}\right)-\frac{1-\pi^{*}}{\pi^{*}} V_{R}^{*}\right\}
$$

Let $\Theta \equiv \frac{\theta}{1-\theta}, \Pi^{*} \equiv \frac{\pi^{*}}{1-\pi^{*}}$ and $J \equiv \frac{V_{I}}{\left(V_{I}+V_{R}^{*}\right)^{2}}\left(\frac{V_{I}+V_{R}^{*}}{2 \Theta}-\frac{V_{R}^{*}}{\Pi^{*}}\right)$. Note that

$$
\frac{\partial J}{\partial V_{R}^{*}}=\frac{-V_{I}}{\left(V_{I}+V_{R}^{*}\right)^{3}}\left\{\left(\frac{1}{2 \Theta}+\frac{1}{\Pi^{*}}\right) V_{I}+\left(\frac{1}{2 \Theta}-\frac{1}{\Pi^{*}}\right) V_{R}^{*}\right\},
$$

and $\pi^{*}<\theta$ as shown by BETT. Then, we find that $\Pi^{*}<2 \Theta$ and $J$ attains at $V_{R}^{*}=\frac{2 \Theta+\Pi^{*}}{2 \Theta-\Pi^{*}} V_{I}$ the unique global minimum of

$$
J_{\min }=-\frac{\left(2 \Theta-\Pi^{*}\right)^{2}}{16 \Pi^{*} \Theta^{2}} \geq-\frac{1-\pi^{*}}{\pi^{*}}
$$

which is the lower bound given in Corollary 2. For the upper bound in (ii), we take the derivative of $\bar{B}^{*}=\frac{1-\pi^{*}}{\pi^{*}} V_{R}^{*}$ with respect to $S$ and get

$$
\frac{d \bar{B}^{*}}{d s}=-\frac{V_{R}^{*}}{\left(\pi^{*}\right)^{2}} \frac{d \pi^{*}}{d s}+\frac{1-\pi^{*}}{\pi^{*}} \frac{d V_{R}^{*}}{d s} .
$$

It can be shown that $\frac{d \pi^{*}}{d s}>0$ if $s$ is uniformly distributed (the proof is available upon request), thus substituting $\bar{B}^{*}$ and $\frac{d \bar{B}^{*}}{d s}$ into (6) we obtain

$$
\frac{d R_{M}^{*}}{d R_{R}^{*}}<\frac{V_{I}}{\left(V_{I}+V_{R}^{*}\right)^{2}}\left\{\frac{1-\pi^{*}}{\pi^{*}}\left(V_{I}+V_{R}^{*}\right)-\frac{1-\pi^{*}}{\pi^{*}} V_{R}^{*}\right\}=\frac{V_{I}^{2}}{\left(V_{I}+V_{R}^{*}\right)^{2}} \frac{1-\pi^{*}}{\pi^{*}}<\frac{1-\pi^{*}}{\pi^{*}}
$$

which provides the upper bound in (ii). 


\section{REFERENCES}

Banerjee, Snehal, 2011, Learning from prices and the dispersion in beliefs, Review of Financial Studies 24, 3025-3068.

Betton, Sandra, B. Espen Eckbo, and Karin S. Thorburn, 2008, Corporate takeovers, in B. E. Eckbo ed.: Handbook of Corporate Finance: Empirical Corporate Finance (Elsevier/North-Holland, Handbooks in Finance Series, Amsterdam).

Betton, Sandra, B. Espen Eckbo, Rex Thompson, and Karin S. Thorburn, 2014, Merger negotiations with stock market feedback, Journal of Finance 69, 1705-1745.

Chordia, Tarun, Avanidhar Subrahmanyam, 2004, Order imbalance and individual stock returns: Theory and evidence, Journal of Financial Economics 72, 485-518.

Hong, Harrison, Jeremy C. Stein, 1999, A unified theory of underreaction, momentum trading, and overreaction in asset markets, Journal of Finance 54, 2143-2184.

Hong, Harrison, Terence Lim, Jeremy C. Stein, 2000, Bad news travels slowly: Size, analyst coverage, and the profitability of momentum strategies, Journal of Finance 55, 265-295.

Jarrell, Gregg A. and Annette Poulsen, 1989, Stock trading before the announcement of tender offers: Insider trading or market anticipation? Journal of Law, Economics, and Organization 50, 225-248.

Pound, John and Richard Zeckhauser, 1990, Clearly heard on the street: The effect of takeover rumors on stock prices, Journal of Business 63, 291-308.

Schwert, G. William, 1996, Markup pricing in mergers and acquisitions, Journal of Financial Economics 41, 153-192.

Zivney, Terry L., William J. Bertin and Khalil M. Torabzadeh, 1996, Overreaction to takeover speculation, Quarterly Review of Economics and Finance 36, 89-115.

[Type text] 
Panel A: Relationship between the rates of runup and markup $38 \%$

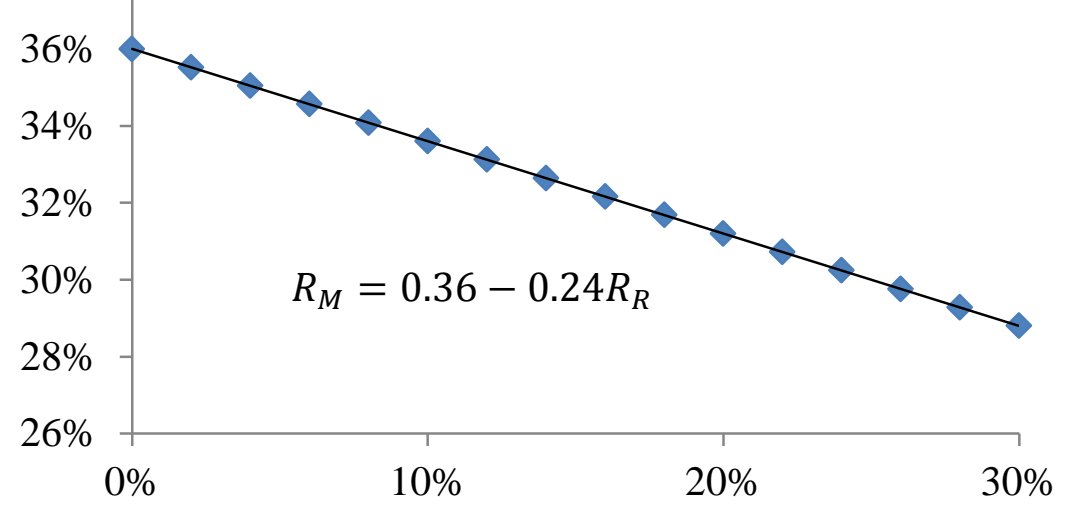

Panel B: Relationship between the values of runup and markup 0.38

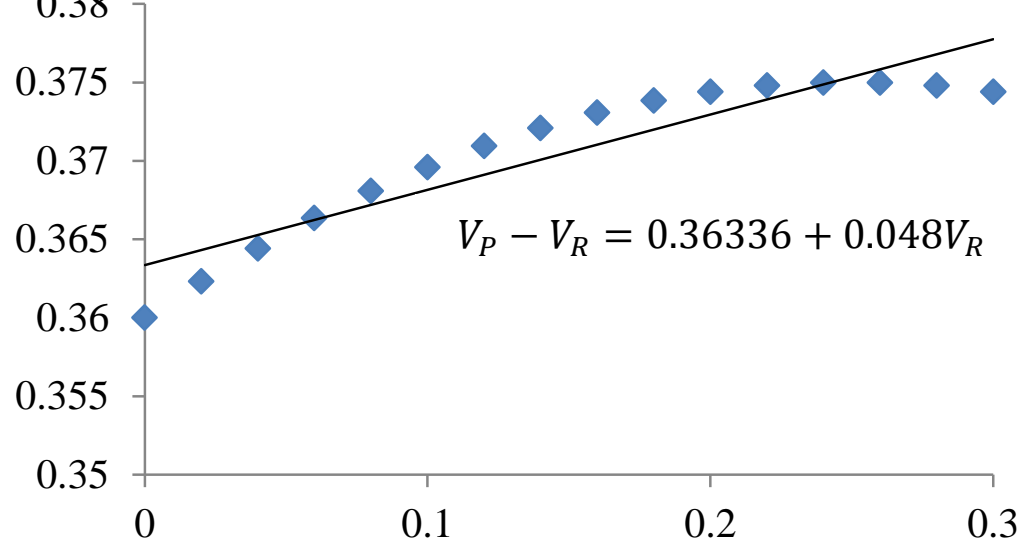

Figure 1. The relationship between the rates of runup and markup vs. the relationship between the values of runup and markup. The data in Panel A are generated from model (1) in Table IV of BETT, such that $R_{M}=0.36-0.24 R_{R}$ for $R_{R i}=$ $0,2 \%, \ldots, 30 \%$, which yields a positive relationship between runup and markup in dollar terms, such that $V_{P}-V_{R}=0.36336+$ $0.048 V_{R}$ in Panel B. The initial value of the target is set at $V_{I}=1$. 

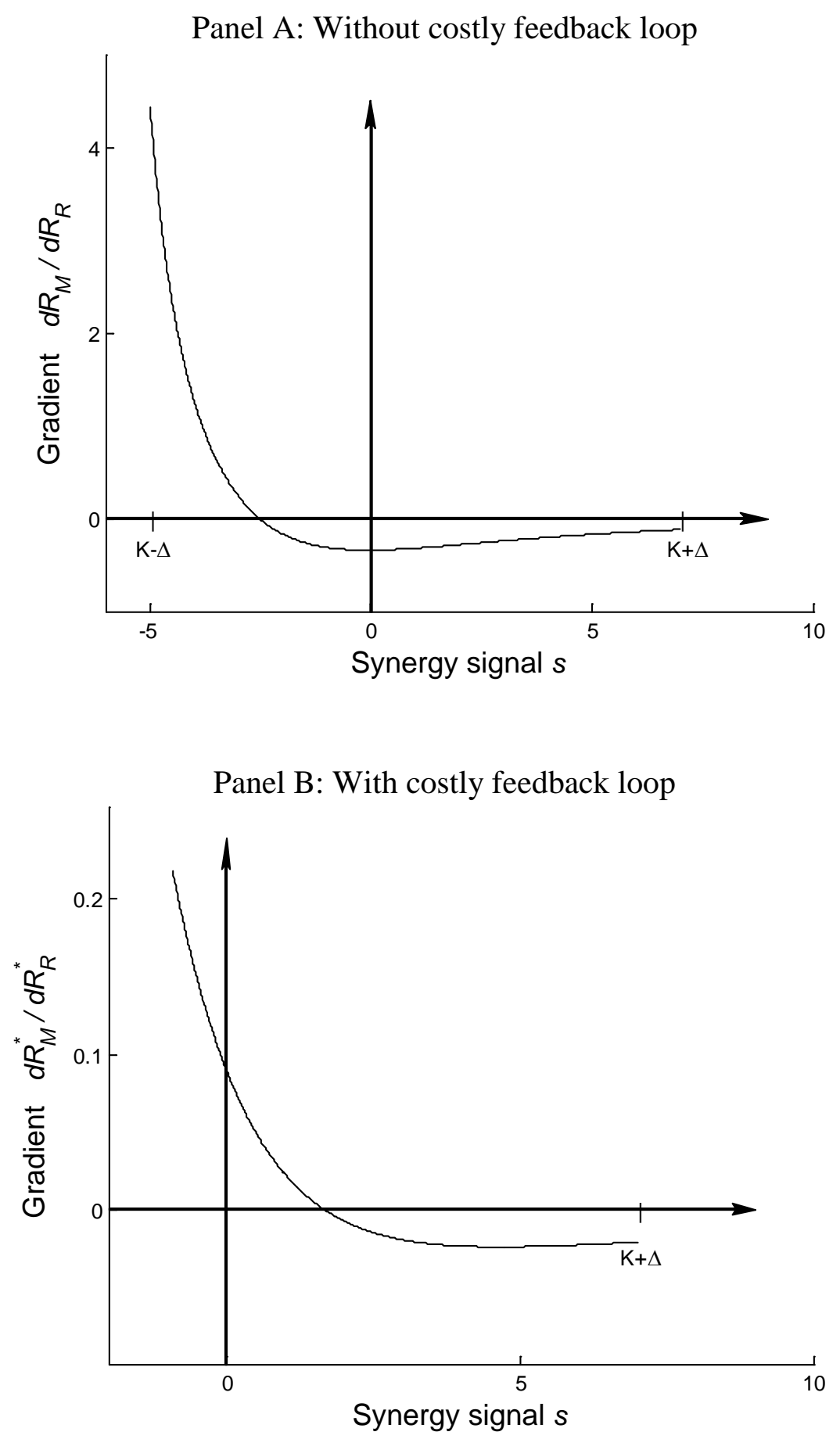

Figure 2. The change in markup rate over runup rate as the synergy signal increases. This figure plots the change in markup rate over runup rate as the synergy signal increases. The market receives a signal $s$ about a potential takeover with synergy $S$, where $S \mid s \sim U(s-\Delta, s+\Delta)$ and $\Delta=6$. The synergy sharing rule is $\theta=0.5$ and bid costs are $C=0.5, K=C / \theta$. In the vertical axis, $R_{M}$ or $R_{M}^{*}$ is the rate of markup and $R_{R}$ or $R_{R}^{*}$ is the rate of runup. The initial value of the target is $V_{I}=1$. 
Table I

Annual Distributions of the Initial Offer Premium, Markup and Runup

The upper panel of this table shows the means and medians of the rate of offer premium, the rate of markup, the rate of runup (which is equal to the standardized runup) and the standardized markup from 1980 through 2013, where $O P$ is the price per share offered by the initial bidder and $P_{t}$ is the target stock price on trading day $t$ relative to the takeover announcement day $(t=0)$. The lower panel reports the standard deviations of the four variables over the two sample periods.

\begin{tabular}{|c|c|c|c|c|c|c|c|c|c|}
\hline \multirow[b]{2}{*}{ Year } & \multirow{2}{*}{$\begin{array}{c}\begin{array}{c}\text { Sample } \\
\text { size }\end{array} \\
\mathrm{N}\end{array}$} & \multicolumn{2}{|c|}{$\begin{array}{l}\text { Rate of offer } \\
\text { premium } \\
\frac{O P}{P_{-42}}-1\end{array}$} & \multicolumn{2}{|c|}{$\begin{array}{l}\text { Rate of markup } \\
\qquad \frac{O P}{P_{-2}}-1\end{array}$} & \multicolumn{2}{|c|}{$\begin{array}{l}\text { Rate of runup } \\
\qquad \frac{P_{-2}}{P_{-42}}-1\end{array}$} & \multicolumn{2}{|c|}{$\begin{array}{c}\text { Standardized } \\
\text { markup } \\
\frac{O P-P_{-2}}{P_{-42}}\end{array}$} \\
\hline & & Mean & Median & Mean & Median & Mean & Median & Mean & Median \\
\hline 1980 & 6 & 0.43 & 0.35 & 0.29 & 0.37 & 0.13 & 0.14 & 0.30 & 0.36 \\
\hline 1981 & 39 & 0.54 & 0.45 & 0.39 & 0.33 & 0.11 & 0.07 & 0.43 & 0.33 \\
\hline 1982 & 36 & 0.42 & 0.46 & 0.38 & 0.38 & 0.04 & 0.03 & 0.38 & 0.40 \\
\hline 1983 & 45 & 0.40 & 0.40 & 0.27 & 0.23 & 0.10 & 0.09 & 0.29 & 0.27 \\
\hline 1984 & 87 & 0.38 & 0.34 & 0.32 & 0.27 & 0.05 & 0.06 & 0.33 & 0.28 \\
\hline 1985 & 135 & 0.38 & 0.35 & 0.26 & 0.22 & 0.09 & 0.11 & 0.28 & 0.24 \\
\hline 1986 & 190 & 0.41 & 0.36 & 0.28 & 0.23 & 0.11 & 0.10 & 0.29 & 0.26 \\
\hline 1987 & 228 & 0.35 & 0.32 & 0.31 & 0.26 & 0.05 & 0.06 & 0.29 & 0.28 \\
\hline 1988 & 295 & 0.56 & 0.49 & 0.37 & 0.30 & 0.15 & 0.10 & 0.41 & 0.34 \\
\hline 1989 & 198 & 0.44 & 0.39 & 0.32 & 0.27 & 0.10 & 0.06 & 0.34 & 0.29 \\
\hline 1990 & 106 & 0.49 & 0.42 & 0.47 & 0.42 & 0.03 & -0.002 & 0.46 & 0.47 \\
\hline 1991 & 97 & 0.52 & 0.45 & 0.43 & 0.34 & 0.07 & 0.06 & 0.44 & 0.38 \\
\hline 1992 & 84 & 0.52 & 0.42 & 0.34 & 0.26 & 0.14 & 0.09 & 0.38 & 0.32 \\
\hline 1993 & 126 & 0.45 & 0.38 & 0.35 & 0.33 & 0.09 & 0.05 & 0.36 & 0.34 \\
\hline 1994 & 217 & 0.41 & 0.38 & 0.32 & 0.30 & 0.07 & 0.07 & 0.34 & 0.31 \\
\hline 1995 & 268 & 0.44 & 0.38 & 0.32 & 0.27 & 0.10 & 0.07 & 0.34 & 0.31 \\
\hline 1996 & 302 & 0.38 & 0.32 & 0.28 & 0.24 & 0.09 & 0.05 & 0.30 & 0.25 \\
\hline 1997 & 422 & 0.39 & 0.35 & 0.25 & 0.21 & 0.12 & 0.10 & 0.27 & 0.25 \\
\hline 1998 & 445 & 0.45 & 0.35 & 0.35 & 0.25 & 0.08 & 0.06 & 0.37 & 0.26 \\
\hline 1999 & 519 & 0.54 & 0.45 & 0.36 & 0.30 & 0.15 & 0.11 & 0.39 & 0.34 \\
\hline 2000 & 415 & 0.50 & 0.42 & 0.37 & 0.32 & 0.11 & 0.06 & 0.39 & 0.34 \\
\hline 2001 & 285 & 0.48 & 0.42 & 0.36 & 0.31 & 0.09 & 0.06 & 0.38 & 0.32 \\
\hline 2002 & 165 & 0.45 & 0.39 & 0.35 & 0.31 & 0.08 & 0.03 & 0.37 & 0.32 \\
\hline 2003 & 202 & 0.42 & 0.34 & 0.29 & 0.23 & 0.11 & 0.07 & 0.31 & 0.25 \\
\hline 2004 & 212 & 0.31 & 0.26 & 0.23 & 0.20 & 0.06 & 0.05 & 0.24 & 0.20 \\
\hline 2005 & 246 & 0.30 & 0.27 & 0.25 & 0.21 & 0.05 & 0.04 & 0.25 & 0.22 \\
\hline 2006 & 291 & 0.31 & 0.27 & 0.26 & 0.21 & 0.04 & 0.03 & 0.26 & 0.22 \\
\hline 2007 & 304 & 0.29 & 0.27 & 0.27 & 0.22 & 0.02 & 0.02 & 0.27 & 0.23 \\
\hline 2008 & 195 & 0.32 & 0.29 & 0.42 & 0.35 & -0.06 & -0.05 & 0.38 & 0.32 \\
\hline 2009 & 111 & 0.55 & 0.46 & 0.42 & 0.32 & 0.09 & 0.07 & 0.45 & 0.34 \\
\hline 2010 & 187 & 0.45 & 0.39 & 0.37 & 0.32 & 0.07 & 0.05 & 0.38 & 0.33 \\
\hline 2011 & 176 & 0.42 & 0.35 & 0.36 & 0.31 & 0.06 & 0.04 & 0.36 & 0.32 \\
\hline 2012 & 168 & 0.44 & 0.36 & 0.39 & 0.31 & 0.05 & 0.04 & 0.39 & 0.33 \\
\hline 2013 & 151 & 0.37 & 0.34 & 0.27 & 0.24 & 0.08 & 0.07 & 0.29 & 0.26 \\
\hline $\begin{array}{l}1980- \\
2008\end{array}$ & 6160 & 0.42 & 0.36 & 0.32 & 0.27 & 0.09 & 0.06 & 0.34 & 0.28 \\
\hline $\begin{array}{l}1980- \\
2013\end{array}$ & 6953 & 0.42 & 0.36 & 0.32 & 0.27 & 0.08 & 0.06 & 0.34 & 0.29 \\
\hline \multicolumn{2}{|c|}{ St. Dev., 1980-2008 } & \multicolumn{2}{|c|}{0.435} & \multicolumn{2}{|c|}{0.345} & \multicolumn{2}{|c|}{0.234} & \multicolumn{2}{|c|}{0.347} \\
\hline St. De & $980-2013$ & \multicolumn{2}{|c|}{0.432} & & & \multicolumn{2}{|c|}{0.233} & \multicolumn{2}{|c|}{0.351} \\
\hline
\end{tabular}

[Type text] 


\section{Table II}

Linear Regressions of Markup against Runup

This table reports the regression results of markup on runup. The focus is on the left panel, in which markup and runup are proxied by three sets of variables in their standardized dollar terms. For comparison, the corresponding results of markup rate regressing on runup rate are documented in the right panel. The regressions are conducted for the 1980-2008 and 1980-2013 sample periods. In the table, $O P$ is the price per share offered by the initial bidder, $P_{t}$ is the target stock price on trading day $t$ relative to the takeover announcement day $(t=0), C A R\left(t_{1}, t_{2}\right)$ is the cumulative abnormal return of target stock over $t_{1}$ through $t_{2}$, $S A V_{M}=\frac{P_{1}-P_{-2}}{P_{-42}}-\frac{\beta}{P_{-42}} \sum_{t=-1}^{t=1} m_{t} P_{t-1}$ is the standardized adjusted-markup and $S A V_{R}=\frac{P_{-2}-P_{-42}}{P_{-42}}-\frac{\beta}{P_{-42}} \sum_{t=-41}^{t=-2} m_{t} P_{t-1}$ is the standardized adjusted-runup, where $\beta$ is the market beta of the target firm and $m_{t}$ is market return on day $t$.

\begin{tabular}{|c|c|c|c|c|c|c|}
\hline & \multicolumn{3}{|c|}{ In Standardized Dollar Terms } & \multicolumn{3}{|c|}{ In Rate Terms } \\
\hline & $\begin{array}{c}\text { Markup } \\
W_{P}-W_{R}\end{array}$ & $\begin{array}{c}\text { Runup } \\
W_{R}\end{array}$ & $\begin{array}{c}\text { Regression } \\
W_{P}-W_{R}=a+b W_{R}\end{array}$ & $\begin{array}{l}\text { Markup } \\
R_{M}\end{array}$ & $\begin{array}{c}\text { Runup } \\
R_{R}\end{array}$ & $\begin{array}{c}\text { Regression } \\
R_{M}=\tilde{a}+\tilde{b} R_{R}\end{array}$ \\
\hline $\begin{array}{l}\text { a. } 1980-2008 \\
N=6160\end{array}$ & $\frac{O P-P_{-2}}{P_{-42}}$ & $\frac{P_{-2}}{P_{-42}}-1$ & $\begin{array}{c}a=0.32(t=69.19) \\
b=0.12(t=6.49)\end{array}$ & $\frac{O P}{P_{-2}}-1$ & $\frac{P_{-2}}{P_{-42}}-1$ & $\begin{array}{c}\widetilde{a}=0.34(t=73.18) \\
\widetilde{b}=-0.22(t=-11.9)\end{array}$ \\
\hline $\begin{array}{l}\text { b. } \quad 1980-2013 \\
N=6953\end{array}$ & $\frac{O P-P_{-2}}{P_{-42}}$ & $\frac{P_{-2}}{P_{-42}}-1$ & $\begin{array}{c}a=0.33(t=74.42) \\
b=0.08(t=4.62)\end{array}$ & $\frac{O P}{P_{-2}}-1$ & $\frac{P_{-2}}{P_{-42}}-1$ & $\begin{array}{c}\tilde{a}=0.34(t=78.91) \\
\tilde{b}=-0.23(t=-13.32)\end{array}$ \\
\hline $\begin{array}{l}\text { c. } \quad 1980-2008 \\
N=6160\end{array}$ & $\frac{P_{1}-P_{-2}}{P_{-42}}$ & $\frac{P_{-2}}{P_{-42}}-1$ & $\begin{array}{c}a=0.21(t=63.56) \\
b=0.06(t=4.38)\end{array}$ & $\frac{P_{1}}{P_{-2}}-1$ & $\frac{P_{-2}}{P_{-42}}-1$ & $\begin{array}{c}\tilde{a}=0.22(t=67.72) \\
\tilde{b}=-0.14(t=-11.1)\end{array}$ \\
\hline $\begin{array}{l}\text { d. } 1980-2013 \\
N=6953\end{array}$ & $\frac{P_{1}-P_{-2}}{P_{-42}}$ & $\frac{P_{-2}}{P_{-42}}-1$ & $\begin{array}{c}a=0.23(t=69.69) \\
b=0.03(t=2.66)\end{array}$ & $\frac{P_{1}}{P_{-2}}-1$ & $\frac{P_{-2}}{P_{-42}}-1$ & $\begin{array}{c}\tilde{a}=0.23(t=74.58) \\
\tilde{b}=-0.16(t=-12.61)\end{array}$ \\
\hline $\begin{array}{l}\text { e. } 1980-2008 \\
N=6160\end{array}$ & $S A V_{M}$ & $S A V_{R}$ & $\begin{array}{c}a=0.21(t=64.48) \\
b=0.05(t=3.74)\end{array}$ & $\operatorname{CAR}(-1,1)$ & $C A R(-41,-2)$ & $\begin{array}{c}\tilde{a}=0.21(t=66.87) \\
\tilde{b}=-0.07(t=-5.54)\end{array}$ \\
\hline $\begin{array}{l}\text { f. } \quad 1980-2013 \\
N=6953\end{array}$ & $S A V_{M}$ & $S A V_{R}$ & $\begin{array}{c}a=0.23(t=70.75) \\
b=0.03(t=1.98)\end{array}$ & $C A R(-1,1)$ & $C A R(-41,-2)$ & $\begin{array}{c}\tilde{a}=0.22(t=73.74) \\
\tilde{b}=-0.09(t=-6.82)\end{array}$ \\
\hline
\end{tabular}

